\title{
On the Hessian of the optimal transport potential
}

\author{
STEFÁn INGI VALDIMARSSON
}

\begin{abstract}
We study the optimal solution of the Monge-Kantorovich mass transport problem between measures whose density functions are convolution with a gaussian measure and a log-concave perturbation of a different gaussian measure. Under certain conditions we prove bounds for the Hessian of the optimal transport potential. This extends and generalises a result of Caffarelli.

We also show how this result fits into the scheme of Barthe to prove BrascampLieb inequalities and thus prove a new generalised Reverse Brascamp-Lieb inequality.
\end{abstract}

Mathematics Subject Classification (2000): 49Q20 (primary); 52A40, 44A35 (secondary).

\section{Introduction}

In this note we prove a generalisation of a theorem of Caffarelli from [6] and indicate how it applies to the methods which Barthe has used in [2] to work with Brascamp-Lieb inequalities.

To set things up, suppose we have given two positive measures, $\mu_{f}$ and $\mu_{g}$, on $\mathbb{R}^{n}$ which are absolutely continuous with respect to the Lebesgue measure and whose density functions are $f$ and $g$ respectively. Suppose further that the measures have finite second-order moments and equal (finite) mass.

Then it is a well-known theorem of Brenier, see [4,5] and the book [11], which asserts that there exists a unique positive measure $\pi$ on $\mathbb{R}^{n} \times \mathbb{R}^{n}$ which has marginals $\mu_{f}$ and $\mu_{g}$ such that $\pi$ is a minimiser for

$$
I[\pi]:=\int_{\mathbb{R}^{n} \times \mathbb{R}^{n}}|x-y|^{2} \mathrm{~d} \pi(x, y)
$$

over all measures on $\mathbb{R}^{n} \times \mathbb{R}^{n}$ with these marginals. Furthermore, $\pi$ has the form $\pi=(\mathrm{Id} \times \nabla \phi) \sharp \mu_{f}$ where $\sharp$ denotes the push-forward and $\nabla \phi$ is a uniquely determined gradient of a convex function which pushes $\mu_{f}$ forward to $\mu_{g}$, i.e. $\nabla \phi \sharp \mu_{f}=\mu_{g}$. We call $\phi$ the optimal transportation potential.

Received October 26, 2006; accepted in revised form August 30, 2007. 
The theorem of Caffarelli that we will generalise is then the following:

Theorem 1.1. Suppose $f$ and $g$ are of the form

$$
f=(\operatorname{det} B)^{-\frac{1}{2}} e^{-\pi\left\langle B^{-1} \cdot, \cdot\right\rangle} \quad \text { and } \quad g=C e^{-\pi\left\langle B^{-1} \cdot, \cdot\right\rangle-H}
$$

where $B$ is a positive-definite symmetric linear transformation, $H$ is convex and $C$ is chosen so that $\int \mathrm{d} \mu_{g}=1$. Then the optimal transport potential $\phi$ satisfies

$$
\operatorname{Hess}(\phi, x) \leq I
$$

where I is the identity transformation.

Here and onwards, where appropriate, this inequality is to be understood in the sense of positive definite linear transformations, i.e. $A \leq G$ if and only if $G-A$ is positive semi-definite.

The purpose of this note is to prove the following generalisation:

Theorem 1.2. Suppose $f$ and $g$ are of the form

$$
f=\left(\operatorname{det}\left(B^{-1} G\right)\right)^{\frac{1}{2}} e^{-\pi\left\langle B^{-1} G \cdot, \cdot\right\rangle} * \mu \quad \text { and } \quad g=C e^{-\pi\left\langle B^{-\frac{1}{2}} A^{-1} B^{-\frac{1}{2}} \cdot, \cdot\right\rangle-H}
$$

where $A, G$ and $B$ are positive definite symmetric linear transformations, $\mu$ is a probability measure on $\mathbb{R}^{n}, H$ is convex and $C$ is chosen so that $\int g=1$. Suppose also that $A \leq G$ and $G B=B G$. Then the optimal transport potential $\phi$ satisfies

$$
\operatorname{Hess}(\phi, x) \leq G
$$

Note that we do not assume that $A$ commutes with either $B$ or $G$. Also note that it would be no restriction if we took the exponent in the definition of $g$ to be $-\pi\left\langle B^{-1} G^{-1} \cdot, \cdot\right\rangle-H$ so the linear transformation $A$ is superfluous.

To see this, note that

$$
-\pi\left\langle B^{-\frac{1}{2}} A^{-1} B^{-\frac{1}{2}} \cdot, \cdot\right\rangle-H=-\pi\left\langle B^{-1} G^{-1} \cdot, \cdot\right\rangle-H^{\prime}
$$

where $H^{\prime}=H+\pi\left\langle\left(B^{-\frac{1}{2}} A^{-1} B^{-\frac{1}{2}}-B^{-\frac{1}{2}} G^{-1} B^{-\frac{1}{2}}\right) \cdot, \cdot\right\rangle$ since $B$ and $G$ commute. Since it is known, see for example [9, page 471], that the condition $A \leq G$ is equivalent to the condition $A^{-1} \geq G^{-1}$ which again is equivalent to the condition $B^{-\frac{1}{2}} A^{-1} B^{-\frac{1}{2}} \geq B^{-\frac{1}{2}} G^{-1} B^{-\frac{1}{2}}$ we see that $H^{\prime}$ is convex if $H$ is convex. However, we choose to include $A$ in the definition of $g$ because the case $g=$ $\operatorname{det} A^{-\frac{1}{2}} e^{-\pi\left\langle A^{-1} \cdot, \cdot\right\rangle}$ will be important in Section 3 .

The proof of Theorem 1.2 is the content of Section 2. It follows similar lines as the proof of Caffarelli in [6] but is somewhat more involved.

In Section 3 we use Theorem 1.2 to get results about Brascamp-Lieb and Reverse Brascamp-Lieb inequalities. We now summarise these results. 
Let $B_{j}: \mathbb{R}^{n} \rightarrow \mathbb{R}^{n_{j}}$ be surjective linear transformations for $j=1, \ldots, m$. Assume that $\bigcap_{j=1}^{m} \operatorname{ker} B_{j}=\{0\}$. Let us define the forms

$$
J\left(\left(f_{j}\right)_{j=1}^{m}\right)=\int_{\mathbb{R}^{n}} \prod_{j=1}^{m} f_{j}^{p_{j}}\left(B_{j} x\right) \mathrm{d} x
$$

and

$$
I\left(\left(g_{j}\right)_{j=1}^{m}\right)=\int_{\mathbb{R}^{n}}^{*} \sup \left\{\prod_{j=1}^{m} g_{j}^{p_{j}}\left(y_{j}\right): \sum_{j=1}^{m} p_{j} B_{j}^{*} y_{j}=x, y_{j} \in \mathbb{R}^{n}\right\} \mathrm{d} x .
$$

We consider the inequalities

$$
J\left(\left(f_{j}\right)_{j=1}^{m}\right) \leq F \prod_{j=1}^{m}\left(\int f_{j}\right)^{p_{j}}
$$

and

$$
I\left(\left(g_{j}\right)_{j=1}^{m}\right) \geq E \prod_{j=1}^{m}\left(\int g_{j}\right)^{p_{j}}
$$

and ask what are the optimal values for $E$ and $F$ such that these inequalities hold for all non-negative integrable functions $f_{j}$ and $g_{j}$. In [10], Lieb proved the fundamental result that (1.1) is exhausted by centred gaussians, meaning that the optimal value for $F$ can be computed by considering only $f_{j}$ of the form $e^{-\pi\left\langle A_{j}, \cdot,\right\rangle}$ where $A_{j}$ is a positive definite symmetric linear transformation. By using the well known fact that $\int e^{-\pi\left\langle A_{j} x, x\right\rangle} \mathrm{d} x=\left(\operatorname{det} A_{j}\right)^{-\frac{1}{2}}$ to calculate the integrals in (1.1) we thus get that the best constant is $F=D^{-\frac{1}{2}}$ where

$$
D=\inf _{A_{j}}\left\{\frac{\operatorname{det}\left(\sum_{j=1}^{m} p_{j} B_{j}^{*} A_{j} B_{j}\right)}{\prod_{j=1}^{m}\left(\operatorname{det} A_{j}\right)^{p_{j}}}\right\} .
$$

In [2], Barthe used methods from the theory of optimal transportation to reprove Lieb's result and also at the same time prove the dual result that (1.2) is also exhausted by centred gaussians and that the best constant there is $E=D^{\frac{1}{2}}$.

We wish to extend the results of Barthe to the setting of generalised BrascampLieb inequalities as introduced in Section 8 of [3]. We begin with the following definition:

Definition 1.3. Suppose $G$ is a positive definite symmetric linear transformation and $f$ and $g$ are non-negative functions. We say that

1. $f$ is of class $G$ if $f$ is the convolution of the gaussian $e^{-\pi\langle G, \cdot\rangle}$ with a positive measure and 
2. $g$ is of inverse class $G$ if $g$ has the form

$$
g=e^{-\pi\left\langle G^{-1} \cdot, \cdot\right\rangle-H}
$$

where $H$ is a convex function.

These classes complement each other in the strategy of Barthe as will become clear in Section 3.

We now wish to consider inequalities (1.1) and (1.2) when $f_{j}$ are functions of class $G_{j}$ and $g_{j}$ are of inverse class $G_{j}$. In this case, the inequalities are also exhausted by centred gaussians, restricted to the relevant class.

Specifically, we have the following theorem:

\section{Theorem 1.4.}

1. (Generalised Brascamp-Lieb)

$$
J\left(\left(f_{j}\right)_{j=1}^{m}\right) \leq \frac{1}{\sqrt{D_{\mathbf{G}}}} \prod_{j=1}^{m}\left(\int f_{j}\right)^{p_{j}}
$$

for all $f_{j}$ of class $G_{j}$ and

2. (Generalised Reverse Brascamp-Lieb)

$$
I\left(\left(g_{j}\right)_{j=1}^{m}\right) \geq \sqrt{D_{\mathbf{G}}} \prod_{j=1}^{m}\left(\int g_{j}\right)^{p_{j}}
$$

for all $g_{j}$ of inverse class $G_{j}$

where

$$
D_{\mathbf{G}}=\inf _{A_{j} \leq G_{j}}\left\{\frac{\operatorname{det}\left(\sum_{j=1}^{m} p_{j} B_{j}^{*} A_{j} B_{j}\right)}{\prod_{j=1}^{m}\left(\operatorname{det} A_{j}\right)^{p_{j}}}\right\} .
$$

Remark 1.5. The first part of the theorem has already been seen in [3] but the second part is new. In [3] it is also noted that we have $D_{\mathbf{G}}>0$ if

$$
\sum_{j=1}^{m} p_{j} B_{j}^{*} G_{j} B_{j} \geq B_{l}^{*} G_{l} B_{l}
$$

for all $l=1, \ldots, m$ and in that case we have that $f_{j}(x)=e^{-\pi\left\langle G_{j} x, x\right\rangle}$ and $g_{j}(x)=$ $e^{-\pi\left\langle G_{j}^{-1} x, x\right\rangle}$ are extremisers for (1.1) and (1.2) respectively so

$$
D_{\mathbf{G}}=\frac{\operatorname{det}\left(\sum_{j=1}^{m} p_{j} B_{j}^{*} G_{j} B_{j}\right)}{\prod_{j=1}^{m}\left(\operatorname{det} G_{j}\right)^{p_{j}}} .
$$

The proof of Theorem 1.4, which is in Section 3, follows the same steps as Barthe does in [2] but the added ingredient is Theorem 1.2. 
This research forms part of my PhD thesis from the University of Edinburgh. I would like to thank my supervisor Tony Carbery for suggesting this problem and for continuing support. Also I thank Robert McCann and Eric Carlen for bringing the paper [6] to my attention and the anonymous referee for helpful comments.

\section{Proof of Theorem 1.2}

First of all, let us note that it is straightforward to verify that the hypotheses of Brenier's Theorem are satisfied so that the potential $\phi$ is indeed defined.

We wish to prove that $\operatorname{Hess}(\phi, x) \leq G$. However, for technical reasons which will become clear, we will make a couple of modifications. First of all, we replace $g$ by $g^{r}: \bar{B}_{r} \mapsto \mathbb{R}$ given by $g^{r}(x)=C^{\prime} g(x)$ for $|x| \leq r$ where $\bar{B}_{r}$ denotes the closed ball in $\mathbb{R}^{n}$ of radius $r$ and $C^{\prime}$ is a normalising constant chosen so that $\int g^{r}=1$. We specify that the function which transports $f \mathrm{~d} \mathcal{L}^{n}$ to $\left.g^{r} \mathrm{~d} \mathcal{L}^{n}\right|_{\bar{B}_{r}}$ is $\nabla \phi^{r}$ where $\phi^{r}$ is convex. Secondly, we replace the Hessian by a finite difference quotient

$$
\frac{\phi^{r}(x+h \alpha)+\phi^{r}(x-h \alpha)-2 \phi^{r}(x)}{h^{2}}
$$

for some fixed $h>0$.

We are therefore interested in the function

$$
K(x, \alpha):=\langle G \alpha, \alpha\rangle-\frac{\phi^{r}(x+h \alpha)+\phi^{r}(x-h \alpha)-2 \phi^{r}(x)}{h^{2}} .
$$

Since $H$ is convex and therefore locally Lipschitz continuous we can see that both $f$ and $g^{r}$ are Lipschitz continuous. Furthermore, $f$ is bounded on $\mathbb{R}^{n}$ and locally bounded away from zero and $g^{r}$ is supported on a convex set and bounded and bounded away from zero there. Then from Caffarelli's regularity theory, it follows that $\phi^{r}$ is $C^{2}$. The relevant theorem is reported in [1], see also [11, Chapter 4]. Thus $K$ is $C^{2}$ on $\mathbb{R}^{n} \times S^{n-1}$ and we wish to show that it is non-negative.

Our strategy will be to show that at any point where $K$ has a local minimum then $K$ is non-negative. From the convexity of $\phi^{r}$ it is clear that

$$
K(x, \alpha) \leq\langle G \alpha, \alpha\rangle
$$

for any $\alpha$ in $S^{n-1}$. We show at the end of this section that in the limit as $x$ tends to infinity this inequality becomes an equality so it is guaranteed that $K$ has a local minimum which is also a global minimum.

If we work with $g$ and $\phi$ directly we cannot hope that (2.1) becomes an equality in the limit as can be easily seen from the example

$$
f=(\operatorname{det} G)^{\frac{1}{2}} e^{-\pi\langle G, \cdot\rangle} \quad g=(\operatorname{det} A)^{-\frac{1}{2}} e^{-\pi\left\langle A^{-1}, \cdot\right\rangle}
$$

with $A$ and $G$ commuting where it is easy to confirm that the transport map is given by

$$
\nabla \phi(x)=A^{\frac{1}{2}} G^{\frac{1}{2}} x
$$


so $\operatorname{Hess}(\phi, x)=A^{\frac{1}{2}} G^{\frac{1}{2}}$ for all $x$ and if $A<G$ this does not equal $G$. So the reason why we use $g^{r}$ instead of $g$ is that the resulting $\phi^{r}$ has much better behaviour at infinity than we can expect of $\phi$ as we shall see in Lemma 2.1 at the end of this section and in the discussion thereafter.

However, we have only been able to prove this good behaviour of $\phi^{r}$ at infinity for the finite difference quotient and not the Hessian itself. That is one reason why we use the finite difference but another is that if we use the finite difference then we only need to know that $\phi^{r}$ is $C^{2}$.

Then we get the pointwise Monge-Ampère equation which is the key equation relating $\phi^{r}$ to $f$ and $g^{r}$;

$$
g^{r}\left(\nabla \phi^{r}(x)\right) \operatorname{det}\left(\operatorname{Hess}\left(\phi^{r}, x\right)\right)=f(x) .
$$

Let us now assume that $K$ has a local minimum at $\left(x_{0}, \alpha_{0}\right)$. We have that

$$
\begin{aligned}
\partial_{x_{i}} K\left(x_{0}, \alpha_{0}\right) & =\partial_{x_{i}}\left(-\frac{\phi^{r}\left(x_{0}+h \alpha_{0}\right)+\phi^{r}\left(x_{0}-h \alpha_{0}\right)-2 \phi^{r}\left(x_{0}\right)}{h^{2}}\right) \\
& =-\frac{\phi_{i}^{r}\left(x_{0}+h \alpha_{0}\right)+\phi_{i}^{r}\left(x_{0}-h \alpha_{0}\right)-2 \phi_{i}^{r}\left(x_{0}\right)}{h^{2}}
\end{aligned}
$$

and since $\left(x_{0}, \alpha_{0}\right)$ is a local minimum we get that

$$
\phi_{i}^{r}\left(x_{0}+h \alpha_{0}\right)+\phi_{i}^{r}\left(x_{0}-h \alpha_{0}\right)-2 \phi_{i}^{r}\left(x_{0}\right)=0 .
$$

Since this holds for $i=1, \ldots, n$ we get that

$$
\nabla \phi^{r}\left(x_{0}+h \alpha_{0}\right)+\nabla \phi^{r}\left(x_{0}-h \alpha_{0}\right)-2 \nabla \phi^{r}\left(x_{0}\right)=0 .
$$

Also, we calculate

$$
\partial_{\alpha^{\perp}} K\left(x_{0}, \alpha_{0}\right)=2 G \alpha_{0} \cdot \alpha^{\perp}-\frac{\nabla \phi^{r}\left(x_{0}+h \alpha_{0}\right) \cdot h \alpha^{\perp}-\nabla \phi^{r}\left(x_{0}-h \alpha_{0}\right) \cdot h \alpha^{\perp}}{h^{2}}
$$

where $\partial_{\alpha^{\perp}}$ denotes the directional derivative in the direction of $\alpha^{\perp}$. Since $\left(x_{0}, \alpha_{0}\right)$ is a local minimum we get that

$$
\left(2 G \alpha_{0}-\frac{\nabla \phi^{r}\left(x_{0}+h \alpha_{0}\right)-\nabla \phi^{r}\left(x_{0}-h \alpha_{0}\right)}{h}\right) \cdot \alpha^{\perp}=0
$$

for any unit vector $\alpha^{\perp}$ which is perpendicular to $\alpha_{0}$. We can interpret this as saying that there exists a $\lambda \in \mathbb{R}$ such that

$$
G \alpha_{0}=\frac{\nabla \phi^{r}\left(x_{0}+h \alpha_{0}\right)-\nabla \phi^{r}\left(x_{0}-h \alpha_{0}\right)}{2 h}-\lambda \alpha_{0} .
$$

Solving this equation together with (2.3) gives

$$
\nabla \phi^{r}\left(x_{0} \pm h \alpha_{0}\right)=\nabla \phi^{r}\left(x_{0}\right) \pm h\left(G \alpha_{0}+\lambda \alpha_{0}\right) .
$$


Let us now take the relevant finite difference in (2.2). This gives

$$
\begin{aligned}
& \log \operatorname{det}\left(\operatorname{Hess}\left(\phi^{r}, x_{0}+h \alpha_{0}\right)\right)+\log \operatorname{det}\left(\operatorname{Hess}\left(\phi^{r}, x_{0}-h \alpha_{0}\right)\right) \\
& -2 \log \operatorname{det}\left(\operatorname{Hess}\left(\phi^{r}, x_{0}\right)\right) \\
& =\log f\left(x_{0}+h \alpha_{0}\right)+\log f\left(x_{0}-h \alpha_{0}\right)-2 \log f\left(x_{0}\right) \\
& -\left[\log g^{r}\left(\nabla \phi^{r}\left(x_{0}+h \alpha_{0}\right)\right)+\log g^{r}\left(\nabla \phi^{r}\left(x_{0}-h \alpha_{0}\right)\right)\right. \\
& \left.\quad-2 \log g^{r}\left(\nabla \phi^{r}\left(x_{0}\right)\right)\right] .
\end{aligned}
$$

Dealing with the individual terms of this equation will be our main task in what follows. We know that log det is a concave function so the graph of the tangent plane at any point lies above the graph of the function. If we use this at $\operatorname{Hess}\left(\phi^{r}, x_{0}\right)$ we get that the left hand side of the equation is less than

$$
\mathrm{D}(\log \operatorname{det})\left(\operatorname{Hess}\left(\phi^{r}, x_{0}\right)\right) \cdot E
$$

where

$$
E:=\operatorname{Hess}\left(\phi^{r}, x_{0}+h \alpha_{0}\right)+\operatorname{Hess}\left(\phi^{r}, x_{0}-h \alpha_{0}\right)-2 \operatorname{Hess}\left(\phi^{r}, x_{0}\right)
$$

and $\mathrm{D}(\log$ det $)$ is the total derivative of $\log$ det. It is clear that $E$ is the Hessian of the function

$$
x \mapsto \phi^{r}\left(x+h \alpha_{0}\right)+\phi^{r}\left(x-h \alpha_{0}\right)-2 \phi^{r}(x)
$$

which by our assumptions attains a maximum at $x_{0}$ and therefore by the second derivative test we see that $E$ is negative semi-definite. By expanding the determinant by minors and using Cramer's formula we can see that

$$
\mathrm{D}(\log \operatorname{det})\left(\operatorname{Hess}\left(\phi^{r}, x_{0}\right)\right) \cdot E=\sum_{i, j}\left(\left(\operatorname{Hess}\left(\phi^{r}, x_{0}\right)\right)^{-1}\right)_{i j} E_{i j}
$$

Now, for a positive semi-definite symmetric matrix $C$ and a negative semi-definite one $E$ we can write $\sum_{i, j} C_{i j} E_{i j}$ as $\operatorname{tr}(E C)$ and if $(-E)^{\frac{1}{2}}$ and $C^{\frac{1}{2}}$ are the positive semi-definite symmetric square roots of $-E$ and $C$ respectively we can calculate

$$
\begin{aligned}
\operatorname{tr}(E C) & =-\operatorname{tr}\left((-E)^{\frac{1}{2}}(-E)^{\frac{1}{2}} C^{\frac{1}{2}} C^{\frac{1}{2}}\right)=-\operatorname{tr}\left((-E)^{\frac{1}{2}} C^{\frac{1}{2}} C^{\frac{1}{2}}(-E)^{\frac{1}{2}}\right) \\
& =-\operatorname{tr}\left((-E)^{\frac{1}{2}} C^{\frac{1}{2}}\left((-E)^{\frac{1}{2}} C^{\frac{1}{2}}\right)^{T}\right) \leq 0 .
\end{aligned}
$$

This tells us that the term we are working on is non-positive because $E$ as defined above is negative semi-definite and $\left(\operatorname{Hess}\left(\phi^{r}, x_{0}\right)\right)^{-1}$ is positive definite. 
Let us then examine the right hand side of (2.6). The second directional derivative of $\log (f)$ in the direction $\alpha_{0}$ is given by

$$
\frac{f_{\alpha_{0} \alpha_{0}}(x)}{f(x)}-\left(\frac{f_{\alpha_{0}}(x)}{f(x)}\right)^{2} .
$$

We therefore perform the following calculation:

$$
\begin{aligned}
f(x) & =\left(\operatorname{det}\left(B^{-1} G\right)\right)^{\frac{1}{2}} \int e^{-\pi\left\langle B^{-1} G(x-y),(x-y)\right\rangle} \mathrm{d} \mu(y), \\
f_{\alpha_{0}}(x) & =\left(\operatorname{det}\left(B^{-1} G\right)\right)^{\frac{1}{2}} \int-2 \pi\left\langle B^{-1} G \alpha_{0}, x-y\right\rangle e^{-\pi\left\langle B^{-1} G(x-y),(x-y)\right\rangle} \mathrm{d} \mu(y)
\end{aligned}
$$

and

$$
\begin{aligned}
f_{\alpha_{0} \alpha_{0}}(x)= & \left(\operatorname{det}\left(B^{-1} G\right)\right)^{\frac{1}{2}} \int 4 \pi^{2}\left(\left\langle B^{-1} G \alpha_{0}, x-y\right\rangle\right)^{2} e^{-\pi\left\langle B^{-1} G(x-y),(x-y)\right\rangle} \mathrm{d} \mu(y) \\
& -\left(\operatorname{det}\left(B^{-1} G\right)\right)^{\frac{1}{2}} \int 2 \pi\left\langle B^{-1} G \alpha_{0}, \alpha_{0}\right\rangle e^{-\pi\left\langle B^{-1} G(x-y),(x-y)\right\rangle} \mathrm{d} \mu(y) .
\end{aligned}
$$

The Cauchy-Schwarz inequality gives us that

$$
\begin{aligned}
\left(f_{\alpha_{0}}(x)\right)^{2}= & \left(\operatorname{det}\left(B^{-1} G\right)\right)\left(\int-2 \pi\left\langle B^{-1} G \alpha_{0}, x-y\right\rangle e^{-\pi\left\langle B^{-1} G(x-y),(x-y)\right\rangle} \mathrm{d} \mu(y)\right)^{2} \\
\leq & \left(\operatorname{det}\left(B^{-1} G\right)\right)^{\frac{1}{2}} \int e^{-\pi\left\langle B^{-1} G(x-y),(x-y)\right\rangle} \mathrm{d} \mu(y) \\
& \cdot\left(\operatorname{det}\left(B^{-1} G\right)\right)^{\frac{1}{2}} \int 4 \pi^{2}\left(\left\langle B^{-1} G \alpha_{0}, x-y\right\rangle\right)^{2} e^{-\pi\left\langle B^{-1} G(x-y),(x-y)\right\rangle} \mathrm{d} \mu(y)
\end{aligned}
$$

and this tells us that

$$
\frac{f_{\alpha_{0} \alpha_{0}}(x)}{f(x)}-\left(\frac{f_{\alpha_{0}}(x)}{f(x)}\right)^{2} \geq-2 \pi\left\langle B^{-1} G \alpha_{0}, \alpha_{0}\right\rangle .
$$

By integrating twice we can estimate the terms involving $f$ from below by

$$
-2 \pi h^{2}\left\langle B^{-1} G \alpha_{0}, \alpha_{0}\right\rangle .
$$

The terms in (2.6) involving $g^{r}$ can be split into a sum of three parts. The first part comes from the normalising constant $C$ and this will equal $2 C-2 C=0$. The third part will be

$$
\begin{gathered}
H\left(\nabla \phi^{r}\left(x_{0}+h \alpha_{0}\right)\right)+H\left(\nabla \phi^{r}\left(x_{0}-h \alpha_{0}\right)\right)-2 H\left(\nabla \phi^{r}\left(x_{0}\right)\right) \\
\geq \operatorname{DH}\left(\nabla \phi^{r}\left(x_{0}\right)\right) \cdot\left(\nabla \phi^{r}\left(x_{0}+h \alpha_{0}\right)+\nabla \phi^{r}\left(x_{0}-h \alpha_{0}\right)-2 \nabla \phi^{r}\left(x_{0}\right)\right)=0
\end{gathered}
$$

where we have used the convexity of $H$ and the condition (2.3). 
The second part is

$$
\begin{aligned}
& \pi\left\langle B^{-\frac{1}{2}} A^{-1} B^{-\frac{1}{2}} \nabla \phi^{r}\left(x_{0}+h \alpha_{0}\right), \nabla \phi^{r}\left(x_{0}+h \alpha_{0}\right)\right\rangle \\
& +\pi\left\langle B^{-\frac{1}{2}} A^{-1} B^{-\frac{1}{2}} \nabla \phi^{r}\left(x_{0}-h \alpha_{0}\right), \nabla \phi^{r}\left(x_{0}-h \alpha_{0}\right)\right\rangle \\
& \quad-2 \pi\left\langle B^{-\frac{1}{2}} A^{-1} B^{-\frac{1}{2}} \nabla \phi^{r}\left(x_{0}\right), \nabla \phi^{r}\left(x_{0}\right)\right\rangle .
\end{aligned}
$$

Using (2.5) we can simplify this to

$$
2 \pi h^{2}\left\langle B^{-\frac{1}{2}} A^{-1} B^{-\frac{1}{2}}\left(G \alpha_{0}+\lambda \alpha_{0}\right), G \alpha_{0}+\lambda \alpha_{0}\right\rangle .
$$

When we take all these calculations together we see that we have reduced (2.6) to the simple inequality

$$
0 \geq-2 \pi h^{2}\left\langle B^{-1} G \alpha_{0}, \alpha_{0}\right\rangle+2 \pi h^{2}\left\langle A^{-1} B^{-\frac{1}{2}}\left(G \alpha_{0}+\lambda \alpha_{0}\right), B^{-\frac{1}{2}}\left(G \alpha_{0}+\lambda \alpha_{0}\right)\right\rangle
$$

which says

$$
\left\langle B^{-1} G \alpha_{0}, \alpha_{0}\right\rangle \geq\left\langle A^{-1} B^{-\frac{1}{2}}\left(G \alpha_{0}+\lambda \alpha_{0}\right), B^{-\frac{1}{2}}\left(G \alpha_{0}+\lambda \alpha_{0}\right)\right\rangle .
$$

We now use the fact that $A^{-1} \geq G^{-1}$, which, as we have already mentioned, follows from the condition $G \geq A$. Using this we see that

$$
\left\langle B^{-1} G \alpha_{0}, \alpha_{0}\right\rangle \geq\left\langle G^{-1} B^{-\frac{1}{2}}\left(G \alpha_{0}+\lambda \alpha_{0}\right), B^{-\frac{1}{2}}\left(G \alpha_{0}+\lambda \alpha_{0}\right)\right\rangle .
$$

and by expanding the right hand side we get that

$$
0 \geq 2 \lambda\left\langle B^{-1} \alpha_{0}, \alpha_{0}\right\rangle+\lambda^{2}\left\langle B^{-1} G^{-1} \alpha_{0}, \alpha_{0}\right\rangle
$$

where we have used the assumption that $B$ and $G$ commute. This is a quadratic expression in $\lambda$ and since both coefficients are positive we can deduce from this that $\lambda \leq 0$. By taking the inner product of (2.4) with $\alpha_{0}$ and using this we get that

$$
\left\langle G \alpha_{0}, \alpha_{0}\right\rangle \geq \frac{\nabla \phi^{r}\left(x_{0}+h \alpha_{0}\right) \cdot \alpha_{0}-\nabla \phi^{r}\left(x_{0}-h \alpha_{0}\right) \cdot \alpha_{0}}{2 h} .
$$

Unfortunately, when we want to use this equation to tell us something about $K(x, \alpha)$ we are forced to take a less than optimal route. This is because we only have information about the behaviour of $\nabla \phi^{r}$ at $x_{0} \pm h \alpha_{0}$ so the best we can do is to say that

$$
\begin{gathered}
\frac{\phi^{r}\left(x_{0}+h \alpha_{0}\right)+\phi^{r}\left(x_{0}-h \alpha_{0}\right)-2 \phi^{r}\left(x_{0}\right)}{h^{2}} \\
\leq \frac{\nabla \phi^{r}\left(x_{0}+h \alpha_{0}\right) \cdot \alpha_{0}-\nabla \phi^{r}\left(x_{0}-h \alpha_{0}\right) \cdot \alpha_{0}}{h} \leq 2\left\langle G \alpha_{0}, \alpha_{0}\right\rangle
\end{gathered}
$$


so we conclude that

$$
K(x, \alpha) \geq-\left\langle G \alpha_{0}, \alpha_{0}\right\rangle
$$

for all $x \in \mathbb{R}^{n}$ and $\alpha \in S^{n-1}$. In terms of $\phi^{r}$ this says that

$$
\frac{\phi^{r}(x+h \alpha)+\phi^{r}(x-h \alpha)-2 \phi^{r}(x)}{h^{2}} \leq\langle G \alpha, \alpha\rangle+\left\langle G \alpha_{0}, \alpha_{0}\right\rangle .
$$

Since $G$ is positive definite there exists a positive number $M$, the ratio of the largest and smallest eigenvalues of $G$, such that $\left\langle G \alpha_{0}, \alpha_{0}\right\rangle \leq M\langle G \alpha, \alpha\rangle$ for any $\alpha \in S^{n-1}$ so from (2.8) we get that

$$
\frac{\phi^{r}(x+h \alpha)+\phi^{r}(x-h \alpha)-2 \phi^{r}(x)}{h^{2}} \leq(M+1)\langle G \alpha, \alpha\rangle .
$$

We note that this estimate is uniform in $h$. Unfortunately, the estimate misses what we intended to prove by a factor of $M+1$ but we can get around that by iterating. This is the same problem that is encountered in [6] but it is addressed in [7] and we follow that argument here.

Let us assume we have the estimate (2.9) with the factor of $M+1$ replaced by a number $a$ greater than 1, uniformly in $h$. We have until now assumed that $h$ is a fixed positive number but if we temporarily allow it to pass to 0 we get that

$$
\left\langle\operatorname{Hess}\left(\phi^{r}, x\right) \alpha, \alpha\right\rangle \leq a\langle G \alpha, \alpha\rangle
$$

Then we notice that

$$
\begin{gathered}
\phi^{r}\left(x_{0}+h \alpha_{0}\right)+\phi^{r}\left(x_{0}-h \alpha_{0}\right)-2 \phi^{r}\left(x_{0}\right) \\
=\int_{0}^{h}\left(\nabla \phi^{r}\left(x_{0}+t \alpha_{0}\right) \cdot \alpha_{0}-\nabla \phi^{r}\left(x_{0}-t \alpha_{0}\right) \cdot \alpha_{0}\right) \mathrm{d} t
\end{gathered}
$$

and we have two ways of estimating the integrand. Its derivative is

$$
\left\langle\operatorname{Hess}\left(\phi^{r}, x_{0}+t \alpha_{0}\right) \alpha_{0}, \alpha_{0}\right\rangle+\left\langle\operatorname{Hess}\left(\phi^{r}, x_{0}-t \alpha_{0}\right) \alpha_{0}, \alpha_{0}\right\rangle
$$

so by (2.10) we get the upper bound $2 t \cdot a\left\langle G \alpha_{0}, \alpha_{0}\right\rangle$. Also, since the integrand is an increasing function of $t$ we have the bound $2 h \cdot\left\langle G \alpha_{0}, \alpha_{0}\right\rangle$ from (2.7). By replacing the integrand by the better of these we get

$$
\frac{\phi^{r}\left(x_{0}+h \alpha_{0}\right)+\phi^{r}\left(x_{0}-h \alpha_{0}\right)-2 \phi^{r}\left(x_{0}\right)}{h^{2}} \leq \frac{2 a-1}{a}\left\langle G \alpha_{0}, \alpha_{0}\right\rangle .
$$

Now, $a_{1}=M+1, a_{n+1}=\frac{2 a_{n}-1}{a_{n}}$ defines a decreasing sequence tending to 1 and by passing to this limit we get that

$$
\frac{\phi^{r}\left(x_{0}+h \alpha_{0}\right)+\phi^{r}\left(x_{0}-h \alpha_{0}\right)-2 \phi^{r}\left(x_{0}\right)}{h^{2}} \leq\left\langle G \alpha_{0}, \alpha_{0}\right\rangle .
$$


which says that

$$
K\left(x_{0}, \alpha_{0}\right) \geq 0
$$

and this shows that

$$
K(x, \alpha) \geq 0
$$

for all $x \in \mathbb{R}^{n}$ and $\alpha \in S^{n-1}$. By letting $h$ tend to 0 we have thus shown that

$$
\left\langle\operatorname{Hess}\left(\phi^{r}, x\right) \alpha, \alpha\right\rangle \leq\langle G \alpha, \alpha\rangle
$$

and by integration we see that

$$
\frac{\nabla \phi^{r}(x+h \alpha) \cdot \alpha-\nabla \phi^{r}(x-h \alpha) \cdot \alpha}{2 h} \leq\langle G \alpha, \alpha\rangle .
$$

Since $g^{r} \mathrm{~d} x$ tends to $g \mathrm{~d} x$ weakly the stability of the transport map, see e.g. [12, 5.23], gives that

$$
\frac{\nabla \phi(x+h \alpha) \cdot \alpha-\nabla \phi(x-h \alpha) \cdot \alpha}{2 h} \leq\langle G \alpha, \alpha\rangle .
$$

A simple variant of the regularity result from [1] shows that that $\phi$ is twice continuously differentiable so we can take the limit as $h \rightarrow 0$ and get

$$
\langle\operatorname{Hess}(\phi, x) \alpha, \alpha\rangle \leq\langle G \alpha, \alpha\rangle
$$

and this is what we intended to prove.

Let us now prove the lemma we left behind. The proof is identical to the proof of Lemma 4 of [6] and is included for completeness.

Lemma 2.1. For any fixed $r$ we have that

$$
\lim _{|x| \rightarrow \infty}\left|\nabla \phi^{r}(x)-r \frac{x}{|x|}\right|=0 .
$$

Proof. Let us fix the vector $y=\nabla \phi^{r}(x)$ and look at the set

$$
\Gamma_{y}:=\left\{y^{\prime} \in \mathbb{R}^{n}: \operatorname{angle}\left(x, y^{\prime}-y\right) \leq \theta\right\}
$$

where $0<\theta<\frac{\pi}{2}$. This is a cone originating from $y$, pointing in the direction of $x$. Since $\nabla \phi^{r}$ is an optimal transport plan, it is known from [8] that the support of its graph is cyclically monotone. This means that if we take $x_{1}, \ldots, x_{m} \in \mathbb{R}^{n}$ and let $y_{i}=\nabla \phi^{r}\left(x_{i}\right)$ then

$$
\sum_{i=1}^{m}\left|x_{i}-y_{i}\right|^{2} \leq \sum_{i=1}^{m}\left|x_{i}-y_{i-1}\right|^{2}
$$

where we let $y_{0}=y_{m}$. In particular, if we take $y^{\prime}=\nabla \phi^{r}\left(x^{\prime}\right)$ where $y^{\prime} \in \Gamma_{y}$ and apply the inequality to $x, x^{\prime}$ and $y, y^{\prime}$ then we get that

$$
\left\langle x^{\prime}-x, y^{\prime}-y\right\rangle \geq 0
$$


and since angle $\left(x, y^{\prime}-y\right) \leq \theta$ we get that angle $\left(x, x^{\prime}-x\right) \leq \frac{\pi}{2}+\theta$ so the preimage of $\Gamma_{y}$ is contained in the concave cone

$$
\Gamma_{x}:=\left\{x^{\prime} \in \mathbb{R}^{n}: \operatorname{angle}\left(x, x^{\prime}-x\right) \leq \frac{\pi}{2}+\theta\right\} .
$$

Since $\frac{\pi}{2}+\theta<\pi$ we see that the complement of the cone $\Gamma_{x}$ contains a ball around the origin of radius $C_{\theta} x$ where $C_{\theta}>0$ and as $x$ tends to infinity the mass of $f$ on the complement of this ball, and thus on $\Gamma_{x}$, will tend to 0 . We then see that

$$
\left(\inf _{x \in B_{r}} g^{r}(x)\right)\left|\Gamma_{y} \cap B_{r}\right| \leq\left(g^{r} \mathrm{~d} \mathcal{L}^{n}\right)\left(\Gamma_{y} \cap B_{r}\right)=\left(g^{r} \mathrm{~d} \mathcal{L}^{n}\right)\left(\Gamma_{y}\right) \leq\left(f \mathrm{~d} \mathcal{L}^{n}\right)\left(\Gamma_{x}\right)
$$

and by compactness we have that $g^{r}$ is bounded away from 0 on $B_{r}$ so we get that

$$
\lim _{|x| \rightarrow \infty}\left|\Gamma_{y} \cap B_{r}\right|=0 .
$$

Letting $\theta$ tend to $\frac{\pi}{2}$ we get the desired conclusion by the geometry of the problem.

With this lemma in hand we can crudely estimate the finite difference

$$
\frac{\phi^{r}(x+h \alpha)+\phi^{r}(x-h \alpha)-2 \phi^{r}(x)}{h^{2}}
$$

by

$$
\frac{\nabla \phi^{r}(x+h \alpha) \cdot \alpha-\nabla \phi^{r}(x-h \alpha) \cdot \alpha}{2 h}
$$

which tends to 0 as $x$ tends to infinity. Therefore we get

$$
\lim _{x \rightarrow \infty} K(x, \alpha)=\langle G \alpha, \alpha\rangle
$$

and as already mentioned, this guarantees the existence of a global minimiser.

\section{Proof of Theorem 1.4}

Firstly, we note that if $D_{\mathbf{G}}=0$ the theorem has no content so in the following we will assume that $D_{\mathbf{G}}>0$. Let us define

$$
E_{\mathbf{G}}:=\inf \left\{\frac{I\left(\left(f_{j}\right)_{j=1}^{m}\right)}{\prod_{j=1}^{m}\left(\int f_{j}\right)^{p_{j}}}: f_{j} \text { of inverse class } G_{j}\right\}
$$

and

$$
F_{\mathbf{G}}:=\sup \left\{\frac{J\left(\left(f_{j}\right)_{j=1}^{m}\right)}{\prod_{j=1}^{m}\left(\int f_{j}\right)^{p_{j}}}: f_{j} \text { of class } G_{j}\right\} .
$$


Our aim is then to prove that

$$
E_{\mathbf{G}}=\sqrt{D_{\mathbf{G}}} \quad \text { and } \quad F_{\mathbf{G}}=\frac{1}{\sqrt{D_{\mathbf{G}}}}
$$

To begin with, let us define

$$
E_{\mathbf{G}, g}:=\inf \left\{\frac{I\left(\left(f_{j}\right)_{j=1}^{m}\right)}{\prod_{j=1}^{m}\left(\int f_{j}\right)^{p_{j}}}: f_{j} \text { centred gaussian of inverse class } G_{j}\right\}
$$

and

$$
F_{\mathbf{G}, g}:=\sup \left\{\frac{J\left(\left(f_{j}\right)_{j=1}^{m}\right)}{\prod_{j=1}^{m}\left(\int f_{j}\right)^{p_{j}}}: f_{j}=e^{-\pi\left\langle A_{j}, \cdot,\right\rangle} \text { for } A_{j} \leq G_{j}\right\} .
$$

Since the infimum for $E_{\mathbf{G}, g}$ is taken over a smaller class of functions than the infimum for $E_{\mathbf{G}}$ we see that $E_{\mathbf{G}, g} \geq E_{\mathbf{G}}$. Also, by calculating the convolution of $\operatorname{det}\left(G_{j}\right)^{\frac{1}{2}} e^{-\pi\left\langle G_{j}, \cdot\right\rangle}$ with the measure which has density function $\operatorname{det}\left(F_{j}\right)^{\frac{1}{2}} e^{-\pi\left\langle F_{j}, \cdot,\right\rangle}$ where $F_{j}=G_{j}\left(G_{j}-A_{j}\right)^{-1} G_{j}-G_{j}$ we can see that if $A_{j} \leq G_{j}$ then $e^{-\pi\left\langle A_{j} \cdot, \cdot\right\rangle}$ is of class $G_{j}$ so $F_{\mathbf{G}} \geq F_{\mathbf{G}, g}$.

We now state three lemmas which will guide us through the proof.

\section{Lemma 3.1.}

$$
F_{\mathbf{G}, g}=\frac{1}{\sqrt{D_{\mathbf{G}}}}
$$

\section{Lemma 3.2.}

$$
E_{\mathbf{G}, g} F_{\mathbf{G}, g}=1
$$

Lemma 3.3. If $g_{j}$ is of inverse class $G_{j}$ and $f_{j}$ is of class $G_{j}$ then

$$
I\left(g_{1}, \ldots, g_{m}\right) \geq D_{\mathbf{G}} J\left(f_{1}, \ldots, f_{m}\right) .
$$

To see how the result follows, note that by Lemma 3.3 we get that $E_{\mathbf{G}} \geq D_{\mathbf{G}} F_{\mathbf{G}}$ and from that we get the string of inequalities

$$
\sqrt{D_{\mathbf{G}}}=E_{\mathbf{G}, g} \geq E_{\mathbf{G}} \geq D_{\mathbf{G}} F_{\mathbf{G}} \geq D_{\mathbf{G}} F_{\mathbf{G}, g}=\sqrt{D_{\mathbf{G}}}
$$

so we get equality all the way and this gives the theorem.

All that remains is to give a proof of the three lemmas. Note first that centred gaussians of inverse class $G_{j}$ are exactly those of the form $e^{-\pi\left\langle A_{j}^{-1} \cdot, \cdot\right\rangle}$ where $A_{j} \leq$ $G_{j}$ so for the first lemma we take $f_{j}=e^{-\pi\left\langle A_{j} \cdot, \cdot\right\rangle}$. Then

$$
J\left(\left(f_{j}\right)_{j=1}^{m}\right)=\int_{\mathbb{R}^{n}} e^{-\pi \sum_{j=1}^{m} p_{j}\left\langle A_{j} B_{j} x, B_{j} x\right\rangle} \mathrm{d} x=\int_{\mathbb{R}^{n}} e^{-\pi Q(x)} \mathrm{d} x
$$


where

$$
Q(x)=\left\langle\sum_{j=1}^{m} p_{j} B_{j}^{*} A_{j} B_{j} x, x\right\rangle
$$

The fact that $\int e^{-\pi\langle A x, x\rangle} \mathrm{d} x=(\operatorname{det} A)^{-\frac{1}{2}}$ for any positive definite linear transformation $A$ gives

$$
\frac{J\left(\left(f_{j}\right)_{j=1}^{m}\right)}{\prod_{j=1}^{m}\left(\int f_{j}\right)^{p_{j}}}=\left(\frac{\prod_{j=1}^{m}\left(\operatorname{det} A_{j}\right)^{p_{j}}}{\operatorname{det}\left(\sum_{j=1}^{m} p_{j} B_{j}^{*} A_{j} B_{j}\right)}\right)^{\frac{1}{2}}
$$

so $F_{\mathbf{G}, g}=\frac{1}{\sqrt{D_{\mathbf{G}}}}$.

For the second lemma let $g_{j}=e^{-\pi\left\langle A_{j}^{-1} \cdot, \cdot\right\rangle}$. Then $I\left(\left(g_{j}\right)_{j=1}^{m}\right)=\int e^{-\pi R(x)} \mathrm{d} x$ where

$$
R(x)=\inf \left\{\sum_{j=1}^{m} p_{j}\left\langle A_{j}^{-1} x_{j}, x_{j}\right\rangle: x=\sum_{j=1}^{m} p_{j} B_{j}^{*} x_{j} \text { where } x_{j} \in \mathbb{R}^{n_{j}}\right\} .
$$

We recall that the dual of a quadratic form $Q$ is defined by

$$
Q^{*}(x)=\sup \left\{|\langle x, y\rangle|^{2}: Q(y) \leq 1\right\}
$$

It is shown in the proof of Lemma 2 in [2] that $R$ is a quadratic form and that $R=Q^{*}$. Then we see that

$$
\frac{I\left(\left(g_{j}\right)_{j=1}^{m}\right)}{\prod_{j=1}^{m}\left(\int g_{j}\right)^{p_{j}}}=\left(\frac{\prod_{j=1}^{m}\left(\operatorname{det} A_{j}^{-1}\right)^{p_{j}}}{\operatorname{det}(R)}\right)^{\frac{1}{2}}
$$

and since $\operatorname{det} R=\operatorname{det} Q^{*}=(\operatorname{det} Q)^{-1}$ we get that $E_{\mathbf{G}, g} F_{\mathbf{G}, g}=1$.

For the third lemma take $f_{j}$ to be of class $G_{j}$ and $g_{j}$ to be of inverse class $G_{j}$. We may assume that $\int f_{j}=\int g_{j}=1$. Then $f_{j}$ and $g_{j}$ satisfy the conditions of Theorem 1.2 with $B$ as the identity transformation and $A$ and $G$ as $G_{j}$ so from that we get that there exists a $C^{2}$ transport potential $\phi_{j}$ such that

$$
g_{j}\left(\nabla \phi_{j}(x)\right) \operatorname{det}\left(\operatorname{Hess}\left(\phi_{j}, x\right)\right)=f_{j}(x)
$$

and $\operatorname{Hess}\left(\phi_{j}, x\right) \leq G_{j}$ for all $x \in \mathbb{R}^{n_{j}}$. Define $\Theta(y):=\sum_{j=1}^{m} p_{j} B_{j}^{*} \nabla \phi_{j}\left(B_{j} y\right)$. Then the Jacobian of $\Theta$ at $y$ is

$$
\operatorname{det}\left(\sum_{j=1}^{m} p_{j} B_{j}^{*} \operatorname{Hess}\left(\phi_{j}, B_{j} y\right) B_{j}\right)
$$


and this is positive by the assumption that $D_{\mathbf{G}}>0$. We then repeat the calculations from the proof of Lemma 3 in [2]

$$
\begin{aligned}
J\left(\left(f_{j}\right)_{j=1}^{m}\right) & =\int_{\mathbb{R}^{n}} \prod_{j=1}^{m} f_{j}^{p_{j}}\left(B_{j} y\right) \mathrm{d} y \\
& =\int_{\mathbb{R}^{n}} \prod_{j=1}^{m} g_{j}^{p_{j}}\left(\nabla \phi_{j}\left(B_{j} y\right)\right) \prod_{j=1}^{m}\left(\operatorname{det}\left(\operatorname{Hess}\left(\phi_{j}, B_{j} y\right)\right)\right)^{p_{j}} \mathrm{~d} y \\
& \stackrel{(*)}{\leq} \frac{1}{D_{\mathbf{G}}} \int_{\mathbb{R}^{n}} \prod_{j=1}^{m} g_{j}^{p_{j}}\left(\nabla \phi_{j}\left(B_{j} y\right)\right) \operatorname{det}\left(\sum_{j=1}^{m} p_{j} B_{j}^{*} \operatorname{Hess}\left(\phi_{j}, B_{j} y\right) B_{j}\right) \mathrm{d} y \\
& \leq \frac{1}{D_{\mathbf{G}}} \int_{\mathbb{R}^{n}}^{*} \sup _{\substack{\Theta(y)=\\
\sum p_{j} B_{j}^{*} x_{j}}}\left(\prod_{j=1}^{m} g_{j}^{p_{j}}\left(x_{j}\right)\right) \operatorname{det}\left(\sum_{j=1}^{m} p_{j} B_{j}^{*} \operatorname{Hess}\left(\phi_{j}, B_{j} y\right) B_{j}\right) \mathrm{d} y
\end{aligned}
$$

where the added ingredient is that in step $(*)$ we have used that $\operatorname{Hess}(\phi, x) \leq G$ so that the inequality follows from the definition of $D_{\mathbf{G}}$. We can therefore make the change of variables $z=\Theta(y)$ and get

$$
\begin{aligned}
J\left(\left(f_{j}\right)_{j=1}^{m}\right) & \leq \frac{1}{D_{\mathbf{G}}} \int_{\mathbb{R}^{n}}^{*} \sup _{z=\sum_{j=1}^{m} p_{j} B_{j}^{*} x_{j}}\left(\prod_{j=1}^{m} g_{j}^{p_{j}}\left(x_{j}\right)\right) \mathrm{d} z \\
& =\frac{1}{D_{\mathbf{G}}} I\left(\left(g_{j}\right)_{j=1}^{m}\right) .
\end{aligned}
$$

This proves the third lemma.

\section{References}

[1] S. Alesker, S. DAR, and V. Milman, A remarkable measure preserving diffeomorphism between two convex bodies in $\mathbb{R}^{n}$, Geom. Dedicata 74 (1999), 201-212.

[2] F. BARTHE, On a reverse form of the Brascamp-Lieb inequality, Invent. Math. 134 (1998), $335-361$.

[3] J. Bennett, A. CArbery, M. Christ and T. TaO, The Brascamp-Lieb inequalities: finiteness, structure and extremals, Geom. Funct. Anal., to appear.

[4] Y. BRENIER, Décomposition polaire et réarrangement monotone des champs de vecteurs, C. R. Acad. Sci. Paris Sér. I Math. 305 (1987), 805-808.

[5] Y. BRENIER, Polar factorization and monotone rearrangement of vector-valued functions, Comm. Pure Appl. Math. 44 (1991), 375-417.

[6] L. A. CAFFARELLI, Monotonicity properties of optimal transportation and the FKG and related inequalities, Comm. Math. Phys. 214 (2000), 547-563.

[7] L. A. CAFFARELli, Erratum: Monotonicity of optimal transportation and the FKG and related inequalities, Comm. Math. Phys. 214 (2000), 547-563; Comm. Math. Phys. 225 (2002), 449-450.

[8] W. GANGBO and R. J. MCCAnN, The geometry of optimal transportation, Acta Math. 177 (1996), 113-161. 
[9] R. A. Horn and C. R. Johnson, "Matrix Analysis", Cambridge University Press, Cambridge, 1985.

[10] E. H. LIEB, Gaussian kernels have only Gaussian maximizers, Invent. Math. 102 (1990), 179-208.

[11] C. Villani, "Topics in Optimal Transportation", Graduate Studies in Mathematics, Vol. 58, American Mathematical Society, Providence, RI, 2003.

[12] C. Villani, "Optimal Transport, Old and New", 2007, July 18, preprint.

UCLA Mathematics Department Box 951555

Los Angeles, CA 90095-1555

valdimarsson@math.ucla.edu 